\title{
An Adaptive Statistical Time-Frequency Method for Detection of Broken Bars and Bearing Faults in Motors Using Stator Current
}

\author{
Birsen Yazıc1, Member, IEEE, and Gerald B. Kliman, Life Fellow, IEEE
}

\begin{abstract}
It is well known that motor current is a nonstationary signal, the properties of which vary with respect to the time-varying normal operating conditions of the motor. As a result, Fourier analysis makes it difficult to recognize fault conditions from the normal operating conditions of the motor. Time-frequency analysis, on the other hand, unambiguously represents the motor current which makes signal properties related to fault detection more evident in the transform domain. In this paper, we present an adaptive, statistical, time-frequency method for the detection of broken bars and bearing faults. Due to the time-varying normal operating conditions of the motor and the effect of motor geometry on the current, we employ a trainingbased approach in which the algorithm is trained to recognize the normal operating modes of the motor before the actual testing starts. During the training stage, features which are relevant to fault detection are estimated using the torque and mechanical speed estimation. These features are then statistically analyzed and segmented into normal operating modes of the motor. For each mode, a representative and a threshold are computed and stored in a data base to be used as a baseline during the testing stage. In the testing stage, the distance of the test features to the mode representatives are computed and compared with the thresholds. If it is larger than all the thresholds, the measurement is tagged as a potential fault signal. In the postprocessing stage, the testing is repeated for multiple measurements to improve the accuracy of the detection. The experimental results from our study suggest that the proposed method provides a powerful and a general approach to the motor-current-based fault detection.
\end{abstract}

Index Terms - Bearing faults, broken bar detection, motor diagnostics, statistical analysis.

\section{INTRODUCTION}

$\mathbf{F}$ AULT detection based on motor current relies on interpretation of the frequency components in the current spectrum that are related to rotor or bearing asymmetries. However, the current spectrum is influenced by many factors, including electric supply, static and dynamic load conditions, noise, motor geometry, and fault conditions. Therefore, the motor current can be best modeled as a nonstationary random signal. Also, the dependency of motor current on the motor geometry requires a flexible, adaptive approach. For many years,

Paper IPCSD 98-45, presented at the 1997 Industry Applications Society Annual Meeting, New Orleans, LA, October 5-9, and approved for publication in the IEEE TRANSACTIONS ON INDUSTRY APPLICATIONS by the Electric Machines Committee of the IEEE Industry Applications Society. This work was supported by the Electrical Distribution and Control Department, General Electric Company. Manuscript released for publication June 29, 1998.

The authors are with the Corporate Research and Development Center, General Electric Company, Niskayuna, NY 12309 USA

Publisher Item Identifier S 0093-9994(99)02233-1. motor current analysis has been implemented using limited mathematical tools and computer capability. These methods are mostly deterministic and based on Fourier transformation [1], [2]. However, it is well known that Fourier transform techniques are not sufficient to represent nonstationary signals. Moreover, the uncertainty involved in the system requires an adaptive statistical framework to address the problem in an efficient way. In recent years, advancement of statistical signal processing methods have provided efficient and optimal tools to process nonstationary signals. In particular, time-frequency and time-scale transformations provide an optimal mathematical framework for the analysis of time-varying, nonstationary signals [3], [4].

In this paper, we propose an adaptive statistical time-frequency method to detect broken bars and bearing faults. The key idea in the proposed method is to transform motor current into a time-frequency spectrum to capture the time variation of the frequency components and to analyze the spectrum statistically to distinguish fault conditions from the normal operating conditions of the motor. Since each motor has a distinct geometry, we adapt a supervised approach in which the algorithm is trained to recognize the normal operating conditions of the motor prior to actual fault detection.

The method consists of four stages: preprocessing, training, testing, and postprocessing. In the preprocessing stage, the analog data are subjected to typical signal conditioning procedures. During the training stage, the time-frequency spectrum of the current is computed and features relevant to fault conditions are extracted using torque and mechanical speed estimation. Next, the feature space is segmented into the normal operating conditions of the motor, and a set of representatives and thresholds are determined for each normal operating mode. The segmentation is performed by either statistical segmentation or torque estimation. Once the algorithm is trained for all the normal operating conditions, the testing stage starts. In this stage, the time-frequency spectrum of the motor is acquired periodically and the features relevant to the fault conditions are extracted. Next, the distance between the test feature and the representative of each normal operating condition is computed. If the test feature is beyond the threshold of all the normal operating conditions, it is tagged as a potential fault signal. During the postprocessing stage, the testing is repeated a number of times to improve the accuracy of the final decision. 
The remainder of this paper is organized as follows. In Section II, we introduce the time-frequency analysis from a perspective relevant to our discussion. In Section III, we outline our approach and compare it with the existing motor fault detection methods. In Section IV, we introduce the preprocessing and training stages. In Sections V and VI, we discuss the testing and postprocessing stages. In Section VII, we present experimental results. Finally, in Section VIII, we briefly discuss further items of interest in this context and conclude our discussion.

\section{Time-Frequency ANALYsis of Motor CuRrent}

The representation of a signal by means of its Fourier spectrum is essential to solving many problems in applied sciences. However, in some instances, Fourier transform is not the most natural or useful way of representing a signal. For example, we often think of music or speech as a signal in which the spectrum evolves over time in a significant way. We imagine that, at each instant, the ear hears a certain combination of frequencies and that these frequencies are constantly changing. This time evolution of frequencies is not reflected in the Fourier transform. In theory, a signal can be reconstructed from its Fourier transform, but the transform contains information about the frequencies of the signal over all times, instead of showing how the frequencies vary with time. For a given a signal $f$, the standard Fourier transform

$$
F(\omega)=\frac{1}{\sqrt{2 \pi}} \int f(t) e^{i \omega t} d t
$$

gives a representation of the frequency content of $f$ averaged over the entire duration of the signal. Therefore, the information concerning time localization of the frequency content cannot be deduced from $F(\omega)$. As a result, Fourier analysis yields poor results for nonstationary signals, that is, signals whose properties evolve over time.

Time localization of nonstationary signals is typically achieved by the Gabor or short-time Fourier transform [5]. This transform works by first dividing the signal into short consecutive or overlapping portions and then computing the Fourier transform of each portion. The idea is to introduce local frequency parameter so that the local Fourier transform looks at the signal through a window over which the signal is approximately stationary. Such a transform results in a twodimensional description of the signal in time-frequency plane composed of spectral characteristics depending on time.

The mathematical description of short-time Fourier transform is given as follows:

$$
F(\omega, n)=\frac{1}{\sqrt{2 \pi}} \int f(t) g\left(t-n t_{o}\right) e^{-i \omega t} d t
$$

where $g$ is an ideal cut-off function. Dividing the current signal into small portions amounts to multiplying $f$ by a translate of $g$, i.e., $g\left(t-n t_{0}\right)$ where $t_{0}$ is the length of the cutoff interval and $n$ is an integer associated with the signal portion. The parameter $\omega$ is similar to the Fourier frequency and many properties of Fourier transform carry over to the short time Fourier transform. However, analysis here depends critically on the choice of the window $g$. Depending on the signal

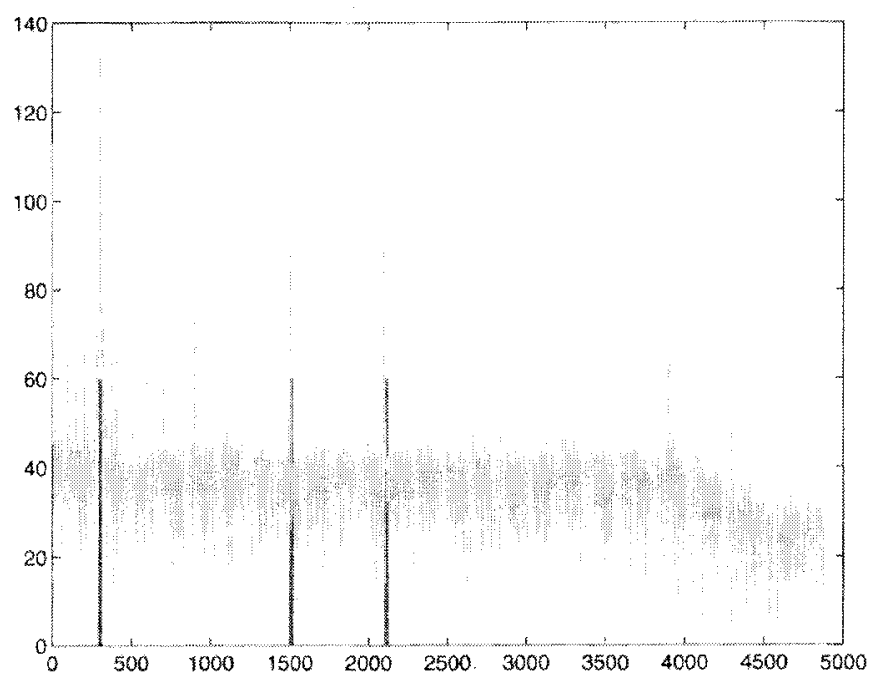

Fig. 1. Fourier spectrum of motor current.

processing task in hand, appropriate choice of the window function $g$ could yield sharp results.

It is well known that motor current is a nonstationary signal. the properties of which vary with respect to the time varying normal operating conditions of the motor, particularly with the load. As a result, Fourier analysis makes it difficult to recognize fault conditions from the normal operating conditions of the motor because Fourier-spectrum-based fault detection implicitly assumes that the motor current becomes nonstationary only in the presence of fault conditions. Time-frequency analysis, on the other hand, unambiguously represents the motor current, which makes signal properties related to parameter estimation and recognition more evident in the transform domain. To illustrate this situation, we acquired motor current from a 3/4-hp motor operating under three different load conditions The motor was first operating under $0.226 \mathrm{~N} \cdot \mathrm{m}(2$ $\mathrm{lb} \cdot \mathrm{in})$ of load torque, then $1.13 \mathrm{~N} \cdot \mathrm{m}(10 \mathrm{lb} \cdot \mathrm{in})$ and then 0.226 $\mathrm{N} \cdot \mathrm{m}(2 \mathrm{lb} \cdot \mathrm{in})$ again. The analog signal was low-pass filtered at $800 \mathrm{~Hz}$ and digitized at 32 samples per $60-\mathrm{Hz}$ voltage supply. As seen in Fig. 1, the Fourier spectrum reveals no information about the load oscillations. The time variations of the frequency content are spread out over the entire frequency range. The time-frequency spectrum shown in Fig. 2, on the other hand, clearly unveils the load oscillations which are important for fault detection. The horizontal axis represents the frequency with $0.2 \mathrm{~Hz} / \mathrm{bin}$ resolution and the vertical axis represents the time with 5-s resolution. The color represents the intensity of the time-frequency spectrum in decibels.

In the following sections, we shall discuss in more detail how time-frequency spectrum can be utilized to devise an efficient fault detection system.

\section{FAUlt DeteCtion SySTEM USING TIME-FREQUENCY TRANSFORM}

In motor-current-based fault detection, there are two main issues:

- how to adapt the detection algorithm to the time varying normal operating conditions of the motor; 


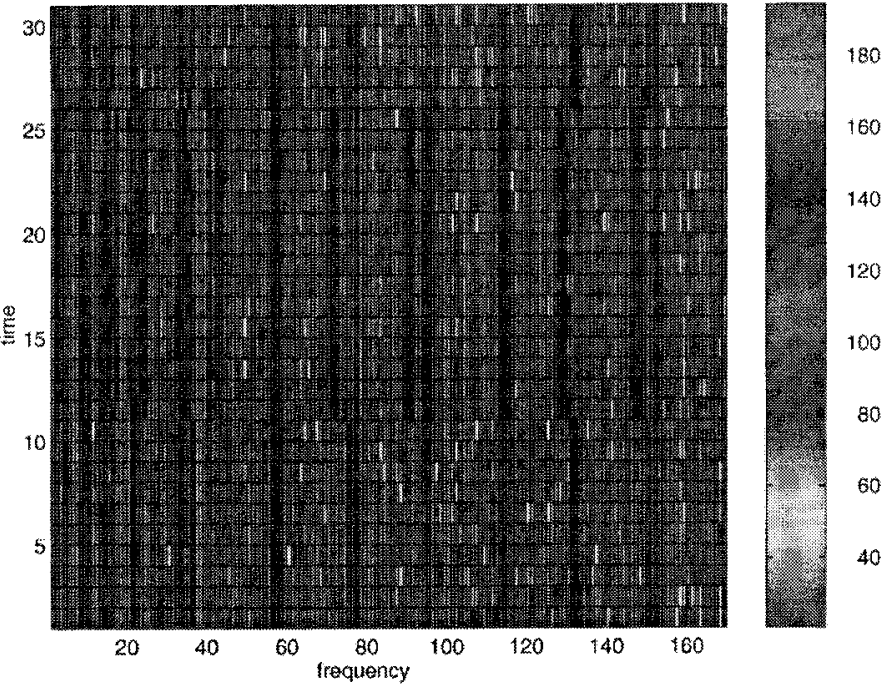

Fig. 2. Time-frequency spectrum of motor current.

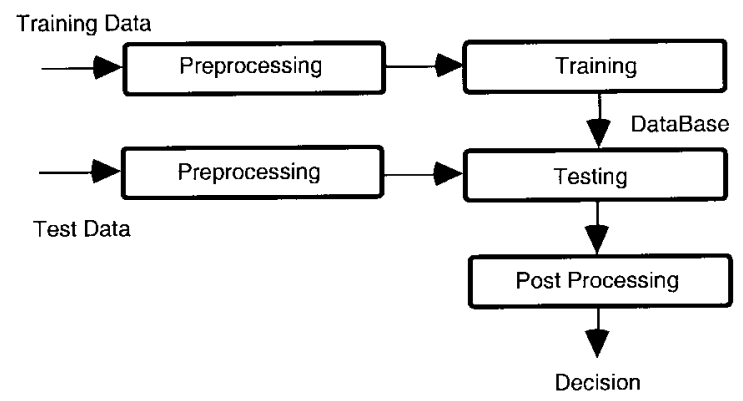

Fig. 3. Block diagram of the fault detection method.

- how to estimate the fault frequencies in the absence of motor geometry and speed information.

The prior methods reported in the literature differ mainly in the way they address these issues. They can be categorized into two classes; although the early methods recognized the nonstationary nature of the motor current, these methods employed Fourier analysis, compromising the accuracy of the detection. In [1], the broken bar frequencies are estimated using speed estimation from the axial leakage flux data. In [6], the frequencies of interest are estimated directly from the current spectrum. With the recent advancement of new signal processing techniques, methods adaptable to time-varying normal operating conditions have been proposed [7]-[9]. These methods utilize rule-based expert systems and neural networks to achieve adaptive detection. However, these operations are performed in the Fourier transform domain, compromising the nonstationary nature of the data.

The block diagram of the proposed fault detection system is shown in Fig. 3. In the preprocessing stage, analog current data are low-pass filtered to prevent aliasing and digitized. Next, the time-frequency spectrum of the data is computed and fed into the training stage. In the training stage, fault frequencies are estimated, and a window of frequency components around the estimated fault frequencies are selected to form a feature vector. Next, feature vectors are segmented into homogenous sections along the time axis in time-frequency space. Segmentation is performed either by a statistical method or by digital torque estimation. Statistical segmentation is a novel method which divides the time-frequency spectrum into statistically homogenous regions along the time axis. Digital torque estimator, on the other hand, divides the motor current into loadwise constant regions. This is a compromise over the statistical segmentation method to reduce the computational load. The homogenous regions obtained from the segmentation are defined as the normal operating modes of the motor. Next, the sample training data belonging to each normal operating mode are statistically analyzed, and a mode representative and a threshold are determined. After all possible normal operating modes of the motor are learned, the testing stage starts. In this stage, the motor current data is periodically acquired and is subjected to the preprocessing and feature extraction methods described in the training stage. The distance of the test feature vector to the representatives of each mode are computed and the resulting distances are compared with the respective thresholds. If any of the distances is larger than the threshold, the test measurement is tagged as a potential fault signal. In the postprocessing stage, the testing process is repeated for a number of measurements to increase the accuracy of the final decision.

The proposed method has the following advantages.

- The method adapts itself to the varying operating conditions of the motor to be tested, thereby offering a more accurate detection of the faults.

- By use of time-varying spectra in the analysis, difficult cases, such as coal crushers where speed varies rapidly, may be efficiently handled.

- Unlike the prior art [2]-[6], the proposed method does not require axial flux leakage data or refined guesses to compute the relevant frequencies. Instead, it utilizes the digital torque estimator [10].

- The proposed method does not require a high-resolution frequency spectrum. The statistical measure adapted at the testing stage takes into account the spread or the resolution of each frequency component. This may reduce the memory required to compute a high-resolution frequency spectrum.

- For the detection of broken bars only, very limited frequency ranges are required, typically within a few slip frequencies around first few harmonics. Consequently, the required computational load is substantially reduced for frequency ranges set by the torque-based estimator.

- Restriction of the frequency range and the knowledge of speed help exclude extraneous components, thus reducing the false alarms when load oscillations and other interference phenomena are present.

- By first identifying and isolating the broken bar and bearing frequency components, they may be excluded from further analysis, thus reducing the computational load and simplifying the analysis of the rest of the frequency components when looking for other types of faults.

- Finally, the proposed framework is applicable to any motor fault which causes rotor asymmetries.

Fig. 4 illustrates the key idea in our fault detection method. 


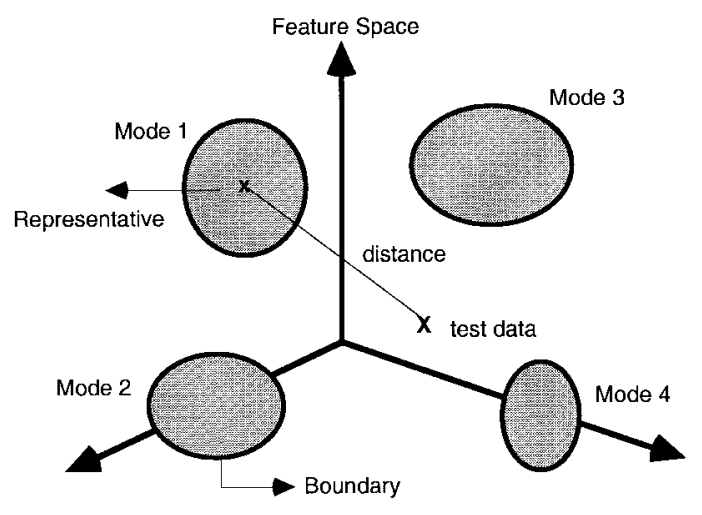

Fig. 4. Three-dimensional illustration of the fault detection method.

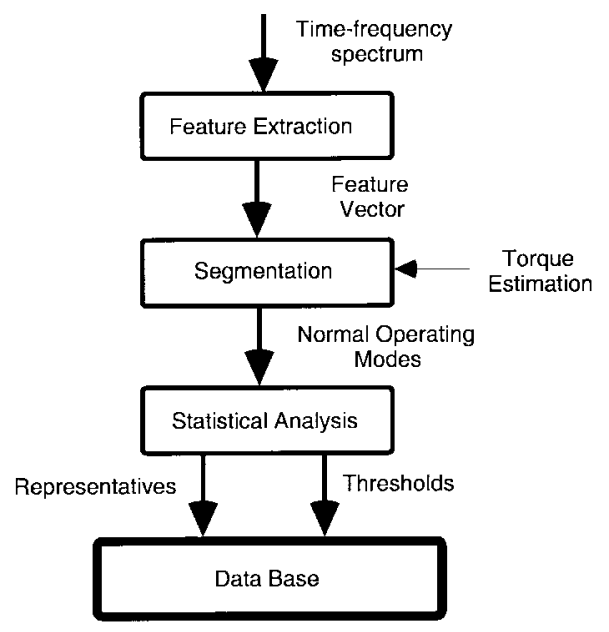

Fig. 5. Block diagram of the training stage.

\section{TRAINING}

The block diagram of the training stage is shown in Fig. 5 . The first step in the training stage is the feature extraction process, in which the frequencies relevant to fault detection are estimated from the time-frequency spectrum. Next, the training features are segmented into the constant operating modes of the motor. Finally, the samples from each mode are statistically analyzed to determine a mode representative and threshold. These values are saved in a data base to be used as a baseline later in the testing stage. Note that the segmentation process and feature extraction process can be interchanged. However, the computational load is much less if the feature extraction is performed prior to segmentation, because the size of the feature vector is much smaller than the size of the entire frequency spectrum.

\section{A. Feature Extraction}

In this section, we shall discuss the estimation of broken bar and bearing fault frequencies. Next, we shall select a window of frequencies around the estimates to form a feature vector. A feature vector is highly informative compressed data which facilitates reliable detection while reducing the computational

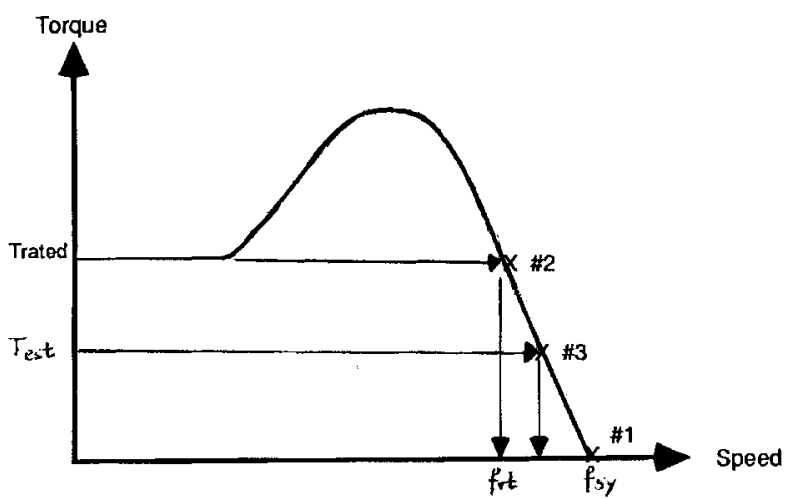

Fig. 6. Torque-speed curve for mechanical speed estimation.

load. We shall call the frequency components associated with the broken bars as the broken bar feature vector, and the frequency components associated with the bearing faults as the bearing feature vector.

In motor theory, it is well known that broken bar faults show up as sideband frequencies of the first, fifth, seventh, and higher order harmonics [1], [7], [11]. These frequencies are given by the following formula:

$$
f_{\mathrm{brk}}=f_{s}\left[k\left(\frac{1-s}{p / 2}\right) \pm s\right], \quad k=1,2,3, \cdots
$$

where $f_{s}$ is the electrical supply frequency, $s$ is per-unit slip, and $p$ is the number of poles. Note that, due to the normal winding conditions, only those frequencies for which $2 k / p=1,5,7,11,13, \cdots$ appear in the stator current with significant amplitude.

From the nameplate of the motor, one can determine the number of poles. However, the slip $s$ changes with the mechanical speed of the motor, which is not readily available. The relationship between the mechanical speed and the slip is given by the following equations:

$$
s=1-\frac{f_{m}}{f_{\mathrm{sy}}}, \quad f_{\mathrm{sy}}=\frac{2 f_{s}}{p}
$$

where $f_{m}$ is the mechanical speed and $f_{\mathrm{sy}}$ is the synchronous speed.

The digital torque estimator provides a simple solution for the estimation of the mechanical speed and the slip frequency [7]-[12]. The technique is illustrated by Fig. 6. The number of poles provides the means for estimating the synchronous speed from torque-speed curve. This estimate is given by point \#1 in Fig. 6. Also, the rated torque and the rated speed of the motor can be obtained from the nameplate, as shown by point \#2. A straight line connecting points 1 and 2 is a sufficient approximation of the motor torque-speed curve in the normal range of loads. The torque estimator finds the actual steadystate motor torque within $2 \%$ error, which is shown by point \#3. The mechanical speed of the motor then can be estimated 
by

$$
f_{m} \cong \frac{T_{\mathrm{est}}}{T_{\mathrm{rt}}}\left(1-\frac{f_{\mathrm{rt}}}{f_{\mathrm{sy}}}\right) f_{s}
$$

where $T_{\text {est }}$ is the estimated torque, $T_{\mathrm{rt}}$ is the rated torque, and $f_{\mathrm{rt}}$ is the rated speed. This is now sufficient to establish the slip frequency within $2 \%$ error.

In the absence of a torque estimator, the broken bar frequencies can be estimated as in [7], which are given by the following formula:

$$
f_{\mathrm{brk}} \cong f_{s} \pm k \cdot f_{m}, \quad k=1,2,3, \cdots .
$$

When the torque estimate is not available, the speed and slip frequency may not be estimated accurately. As a result, the broken bar frequency estimate will be less accurate, since the nameplate provides the speed at full load only.

Rotor asymmetry, resulting from rotor ellipticity, misalignment of the shaft with the cage, magnetic anisotropy, etc., shows up at the same frequency components as the broken bars and must be distinguished from the broken bar frequencies. This can be achieved by examining the sidebands of the higher harmonics. An asymmetry results in low high-frequency content. In contrast, localized effects, such as a broken bar, result in large high-frequency content.

After estimating the broken bar frequencies, a window of frequency components around the estimates is selected to form the broken bar feature vector. Typically, the window is chosen such that at least $0.25 \mathrm{~Hz}$ on each side of the estimate are included in the feature vector. Explicitly, the broken bar feature vector $F_{\mathrm{brk}}(t)$ at time instant $t$ can be written as

$$
\begin{aligned}
F_{\mathrm{brk}}(t)= & {\left[F_{\mathrm{brk}}^{1}(t), F_{\mathrm{brk}}^{5}(t), \cdots\right] } \\
F_{\mathrm{brk}}^{n}(t)= & {\left[S\left(f_{\mathrm{brk}}^{n}-w, t\right), \cdots, S\left(f_{\mathrm{brk}}^{n}, t\right), \cdots,\right.} \\
& \left.S\left(f_{\mathrm{brk}}^{n}+w, t\right)\right], \quad n=1,5,7,11, \cdots
\end{aligned}
$$

where $S$ is the magnitude of the time-frequency representation, i.e., $S(f, t)=|F(f, t)|^{2}$, and $w$ is the length of the window around the estimates $f_{\mathrm{brk}}^{n}, n=1,5,7,11, \cdots$ in $\mathrm{Hz} / \mathrm{bin}$. Note that, for $n=1$, the slip frequency components are on both sides of the supply frequency due to the speed oscillations, but for higher harmonics, they are only on the lower side of the supply frequency.

A bad rolling element bearing in a motor allows the shaft to move radially a small amount. For example, if there is a pit in the outer or the inner race, the balls encountering it will fall in and move radially. Thus, the air-gap geometry will be slightly disturbed, leading to a modulation of the current. These modulation effects will show up at frequencies

$$
f_{\mathrm{brg}}=f_{s} \pm n \cdot f_{v}, \quad n=1,2,3, \cdots
$$

where $f_{v}$ is the mechanical vibration frequency, the value of which depends on the type of the race defect and geometry of the bearing. If the bearings have 6-12 rolling elements, the inner race bearing defect frequencies can be estimated by [13]

$$
f_{\text {brg }}=f_{s} \pm n \cdot 0.6 \cdot f_{m}, \quad n=6,7, \cdots, 12 .
$$

Similarly, the outer race bearing defect frequencies can be estimated by [13]

$$
f_{\mathrm{brg}}=f_{s} \pm n \cdot 0.4 \cdot f_{m}, \quad n=6,7, \cdots, 12
$$

If torque estimation is available, the mechanical speed of the motor can be estimated as described in (4.3), and a window of frequencies around the estimated bearing frequencies

$$
\begin{aligned}
& F_{\mathrm{brg}}(t) \\
& \quad=\left[F_{\mathrm{brg}}^{6}(t), F_{\mathrm{brg}}^{7}(t), \cdots, F_{\mathrm{brg}}^{12}(t)\right] \\
& F_{\mathrm{brg}}^{n}(t) \\
& \quad=\left[S\left(f_{\mathrm{brg}}^{n}-w, t\right), \cdots, S\left(f_{\mathrm{brg}}^{n}, t\right), \cdots, S\left(f_{\mathrm{brg}}^{n}+w, t\right)\right], \\
& \quad n=6,7, \cdots, 12
\end{aligned}
$$

is selected to form the bearing feature vector. Typically, the length of the window is chosen to be at least $1 \mathrm{~Hz}$ on each side of the estimated frequency. If torque estimation is not available, all the frequency components between the rated speed and the synchronous speed can be included in the bearing feature vector.

Finally, the feature vector at time instance $t$ is formed by combining the broken bar and the bearing feature vectors

$$
F(t)=\left[F_{\mathrm{brk}}(t), F_{\mathrm{brg}}(t)\right] .
$$

\section{B. Segmentation}

As discussed earlier, the motor current is a piecewise stationary signal in which different constant operating modes correspond to different statistically homogenous segments. Therefore, it is necessary to determine different baseline thresholds for different operating modes of the motor. To do so, we compute the time-frequency spectrum of the motor for all possible normal operating modes of the motor during the training stage and segment the spectrum into homogenous segments along the time axis. The segmentation process is performed either by a novel probabilistic method or by torque estimation. First, we shall discuss the probabilistic approach.

Let us assume a number of feature vectors $F(t), t=$ $1, \cdots, N$ are available for the training process where $N$ is the total training duration. We assume that the normal operating modes of the motor change slowly and contiguous feature vectors are likely to fall into the same mode. Given these assumptions, the segmentation process can be viewed as the detection of time instants $t_{s}$ at which $F(t), t \leq t_{s}$ and $F(t), t>t_{s}$ exhibit different statistical properties. We shall call this instant split point. To implement the detection process, we divide the feature vectors $F(t), t=1, \cdots, N$ into contiguous windows along the time axis. The length of the time window $T$ is chosen so that the motor can exhibit at most two different operating modes during $T$ units of time. Within each time window, the location of the split point is detected by maximizing the conditional joint probability density function of the feature vectors within the window, given the location 


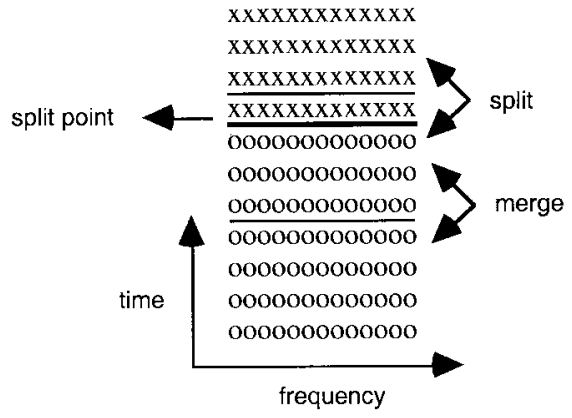

Fig. 7. Probabilistic segmentation for the time-frequency spectrum.

of the split point. This can be mathematically expressed as

$\hat{t}_{s}=\underset{t_{s}=0, \cdots, T-1}{\operatorname{argmax}} \operatorname{Pr}\left(F\left(t_{0}+i\right), \quad i=0, \cdots, T-1 \mid i=t_{s}\right)$

where $t_{0}$ is the beginning of the time window, $t_{s}$ is the candidate location for the split point, and $F\left(t_{0}+i\right), i=$ $0, \cdots, T-1$ are the feature vectors within the time window. Note that $\hat{t}_{s}=T-1$ is interpreted as no mode change during the time window $T$. Once all the time windows are studied and the locations of all the split points are estimated, segments that are similar are merged using the Bhattacharyya distance [14]. Fig. 7 illustrates the probabilistic method of forming different normal operating modes. For more details on the probabilistic segmentation process, please refer to [15].

In the torque-based segmentation, the time instants at which significant load changes occur are recorded and the time-frequency spectrum in each loadwise constant segment is computed. Next, the feature vectors for each segment are computed and utilized to determine a statistical representative and threshold for each loadwise constant mode of the motor. Although this approach is computationally less expensive as compared to the previous one, it cannot identify many of the subtle statistical changes that occur, such as the ones due to shaft misalignments, in the current spectrum. As a result, the accuracy of detection may be less than the previous approach.

\section{Selection of Mode Representatives and Thresholds}

For each constant operating mode of the motor, the sample mean and the covariance matrix of the feature vectors are chosen as the representatives of the mode. Mathematically, these representatives are given by

$$
\begin{aligned}
M_{j} & =\frac{1}{L_{j}} \sum_{k=1}^{L_{j}} F\left(t_{k}\right) \\
C_{j} & =\frac{1}{L_{j}} \sum_{k=1}^{L_{j}}\left(F\left(t_{k}\right)-M_{j}\right)\left(F\left(t_{k}\right)-M_{j}\right)^{T}, \quad j=1, \cdots, L
\end{aligned}
$$

where $L$ is the total number of distinct operating modes of the motor, $L_{j}$ is the number of feature vectors belonging to the mode $j$, and $t_{k}$ is the time instance of a feature vector in a given homogenous mode. We shall refer to the mean and covariance matrix of a mode as mode representative and denote it by

$$
R_{j} \equiv\left(M_{j}, C_{j}\right), \quad j=1, \cdots, L .
$$

Recall that the feature vector $F\left(t_{k}\right)$ is composed of the broken bar feature vector $F_{\mathrm{brk}}\left(t_{k}\right)$ and the bearing feature vector $F_{\mathrm{brg}}\left(t_{k}\right)$. Therefore, the mode representative is composed of broken bar and bearing mode representatives

$$
R_{j}=\left(R_{j}^{\mathrm{brk}}, R_{j}^{\mathrm{brg}}\right), \quad j=1, \cdots, L
$$

in which

$$
R_{j}^{\mathrm{brk}} \equiv\left(M_{j}^{\mathrm{brk}}, C_{j}^{\mathrm{brk}}\right), \quad R_{j}^{\mathrm{brg}} \equiv\left(M_{j}^{\mathrm{brg}}, C_{j}^{\mathrm{brg}}\right)
$$

where

$$
\begin{aligned}
M_{j} & =\left[M_{j}^{\mathrm{brk}}, M_{j}^{\mathrm{brg}}\right] \\
C_{j} & =\left[\begin{array}{cc}
C_{j}^{\mathrm{brk}} & C_{j}^{c} \\
C_{j}^{c} & C_{j}^{\mathrm{brg}}
\end{array}\right]
\end{aligned}
$$

and $C_{j}^{c}$ is the covariance matrix between the broken bar and the bearing feature vectors.

It is important to note that, once the bearing and broken bar mode representatives are obtained, the distance between the distinct operating modes with respect to the broken bars and bearings are calculated and stored in the data base to be used in the postprocessing stage. The Bhattacharyya distance between two modes is given by

$$
\begin{aligned}
B\left(R_{i}, R_{j}\right) \equiv & \frac{1}{8}\left(M_{i}-M_{j}\right)^{T}\left(\frac{C_{i}+C_{j}}{2}\right)^{-1}\left(M_{i}-M_{j}\right) \\
& +\frac{1}{2} \ln \left(\frac{\operatorname{det}\left(\frac{C_{i}+C_{j}}{2}\right)}{\sqrt{\operatorname{det}\left(C_{i} C_{j}\right)}}\right) .
\end{aligned}
$$

Note that the first term in the summation measures the distance in terms of mean values, and the second term measures in terms of the covariance matrices.

We now want to define broken bar and bearing fault thresholds for each normal operating mode of the motor. To do so, we first define a statistical distance between each member of the mode and its representative

$$
\begin{aligned}
D_{j, k}^{\mathrm{brk}} & \equiv d\left(F_{\mathrm{brk}}\left(t_{k}\right), R_{j}^{\mathrm{brk}}\right) \\
& \equiv\left(F_{\mathrm{brk}}\left(t_{k}\right)-M_{j}^{\mathrm{brk}}\right)^{T} C_{j}^{\mathrm{brk}-1}\left(F_{\mathrm{brk}}\left(t_{k}\right)-M_{j}^{\mathrm{brk}}\right) \\
& \quad j=1, \cdots, L, \quad k=1, \cdots, L_{j} .
\end{aligned}
$$

Similarly,

$$
\begin{aligned}
D_{j, k}^{\mathrm{brg}} & \equiv d\left(F_{\mathrm{brg}}\left(t_{k}\right), R_{j}^{\mathrm{brg}}\right) \\
& \equiv\left(F_{\mathrm{brg}}\left(t_{k}\right)-M_{j}^{\mathrm{brg}}\right)^{T} C_{j}^{\mathrm{brg}-1}\left(F_{\mathrm{brg}}\left(t_{k}\right)-M_{j}^{\mathrm{brg}}\right), \\
& \quad j=1, \cdots, L, \quad k=1, \cdots, L_{j} .
\end{aligned}
$$

Note that the distance measure is chosen to be the well-known Mahalanobis distance, in which each entry in the Euclidean distance is downweighted by the variance of the entry [16]. 


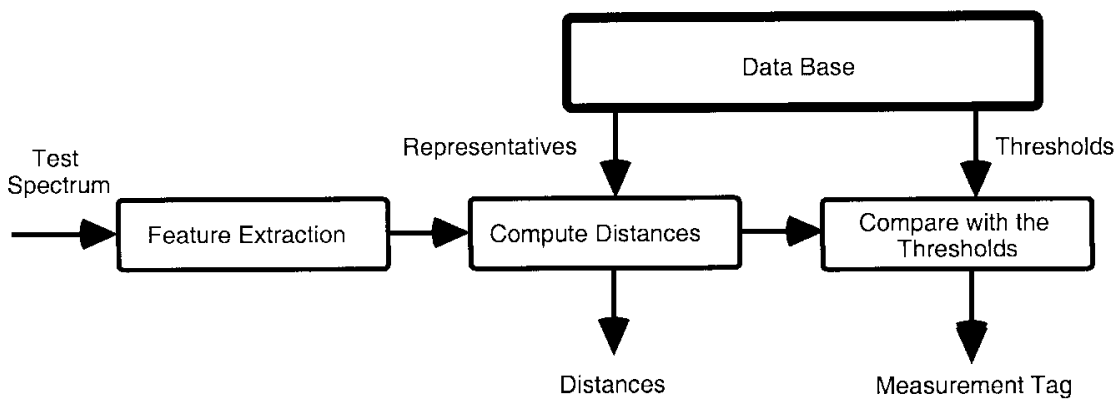

Fig. 8. Block diagram of the testing stage.

In order to obtain an optimal broken bar and bearing fault threshold for each mode, the sample mean and standard deviation of the intramode distances are calculated

$$
\begin{aligned}
\bar{D}_{j}^{\mathrm{brk}} & =\frac{1}{L_{j}} \sum_{k=1}^{L_{j}} D_{j, k}^{\mathrm{brk}} \\
\sigma_{j}^{\mathrm{brk}} & =\sqrt{\frac{1}{L_{j}} \sum_{k=1}^{L_{j}}\left(D_{j, k}^{\mathrm{brk}}-\bar{D}_{j}^{\mathrm{brk}}\right)^{2},} \quad j=1, \cdots, L .
\end{aligned}
$$

Similarly,

$$
\begin{aligned}
\bar{D}_{j}^{\mathrm{brg}} & =\frac{1}{L_{j}} \sum_{k=1}^{L_{j}} D_{j, k}^{\mathrm{brg}} \\
\sigma_{j}^{\mathrm{brg}} & =\sqrt{\frac{1}{L_{j}} \sum_{k=1}^{L_{j}}\left(D_{j, k}^{\mathrm{brg}}-\bar{D}_{j}^{\mathrm{brg}}\right)^{2}}, \quad j=1, \cdots, L .
\end{aligned}
$$

The broken bar and bearing fault thresholds $\tau_{j}^{\mathrm{brk}}$, and $\tau_{j}^{\mathrm{brg}}$ for each operating mode are set $\alpha$ unit standard deviation away from the mean distances, i.e.,

$$
\begin{aligned}
\tau_{j}^{\mathrm{brk}} & =\bar{D}_{j}^{\mathrm{brk}}+\alpha \sigma_{j}^{\mathrm{brk}} \\
\tau_{j}^{\mathrm{brg}} & =\bar{D}_{j}^{\mathrm{brg}}+\alpha \sigma_{j}^{\mathrm{brg}}, \quad j=1, \cdots, L .
\end{aligned}
$$

Note that, in the case of normal distribution of the intramode distances, $\alpha$ is typically chosen to be 2 to provide a $99 \%$ confidence interval. However, in our approach, it is kept as an input parameter to allow the user to utilize one's engineering judgment.

\section{TESTING}

The major steps of the testing stage are illustrated in Fig. 8. Similar to the training process, the test data are first subject to the operations discussed in the preprocessing stage and feature extraction process discussed above. Next, using the Mahalanobis distance introduced in (4.15), the distance between the test feature $F_{t}$ and the representatives of the normal operating modes $R_{j}$ is computed. Depending on whether we want to check the condition of the broken bars or bearings, appropriate portions of the feature vector and representatives are used. For example, in the case of broken bars, the distance is computed as

$$
\Delta_{j}^{\mathrm{brk}}=d\left(F_{t}^{\mathrm{brk}}, R_{j}^{\mathrm{brk}}\right), \quad j=1, \cdots, L .
$$

Next, we check if the distance $\Delta_{j}^{\text {brk }}$ between the test feature and the representatives of each mode is less than the mode threshold $\tau_{j}^{\text {brk }}$. If the distance is greater than all the mode thresholds, $\tau_{j}^{\text {brk }}, j=1, \cdots, L$, then the test measurement is tagged as potentially faulty.

To improve the accuracy of our decision making, we repeat this testing process for multiple test features. The decisions obtained from each test feature and the distances are fed to the postprocessor to finalize the decision on the bars or the bearings of the motor.

\section{POSTPROCESSING}

The assumption in the postprocessing stage is that, if there is a broken bar or bearing fault, consecutive test samples are expected to be potential fault features and will eventually form a mode which is statistically distinct from all the normal operating modes of the motor. Therefore, as the potential fault features are identified by the testing stage, the mean $M_{F}$ and the covariance matrix $C_{F}$ of the potential fault features are computed. Note that these feature vectors could be broken bar or bearing fault feature vectors. Next, the distance $B\left(R_{F}, R_{j}\right)$ between the normal operating modes of the motor and the representatives $\left(M_{F}, C_{F}\right)$ of the faulty features are computed using the Bhattacharyya distance. The normal operating mode to which the potential fault representative is closest is identified, i.e.,

$$
\hat{j}=\underset{j=1, \cdots, L}{\operatorname{argmin}} B\left(R_{F}, R_{j}\right) .
$$

The shortest Bhattacharyya distance, $\Omega_{j}$, of the mode $\hat{j}$ is retrieved from the data base and compared with the $B\left(R_{F}, R_{\hat{j}}\right)$. If the distance $B\left(R_{F}, R_{\hat{j}}\right)$ is greater than the distance $\Omega_{\hat{j}}$, the final alarm for the broken bars or faulty bearings is triggered.

\section{EXPERIMENTAL RESULTS}

For broken bar detection experiments, the raw data from a 35-hp inside-out motor were generated and archived 
TABLE I

Training Data From 3/4-HP Motor with Good Bearing

\begin{tabular}{c||l|l|l|l}
\hline \hline Mode & File Name* & Torque & Speed & \multicolumn{1}{|c}{$\begin{array}{c}\text { Bearing and } \\
\text { data ID }\end{array}$} \\
\hline \hline 1 & H0895001.asc & $-0.339 \mathrm{~N}-\mathrm{m}-$ & $1789 \mathrm{Rpm}$ & $\mathrm{C} 2$ \\
\hline 2 & H0895002.asc & $0.3164 \mathrm{~N}-\mathrm{m}$ & $1790 \mathrm{Rpm}$ & $\mathrm{C} 2$ \\
\hline 3 & H0895003.asc & $+1.356 \mathrm{~N}-\mathrm{m}$ & $1762 \mathrm{Rpm}$ & $\mathrm{C} 2$ \\
\hline 4 & H0895004.asc & $+2.845 \mathrm{~N}-\mathrm{m}$ & $1718 \mathrm{Rpm}$ & $\mathrm{C} 2$ \\
\hline 5 & H0895005.asc & $+1.356 \mathrm{~N}-\mathrm{m}$ & $1762 \mathrm{Rpm}$ & $\mathrm{C} 2$ with turn faults \\
\hline
\end{tabular}

for Electric Power Research Institute (EPRI) Broken Bar Project-EPRI RP2331-1 in 1986 [2]. For bearing fault detection experiments, a 3/4-hp motor was used. In both cases, the analog current data were low-pass filtered at 800 $\mathrm{Hz}$ and digitized at 32 samples per power cycle. However, as we shall discuss, the algorithm does not require frequencies larger than $300 \mathrm{~Hz}$, and sampling frequency can be as low as six samples per power cycle. Each data file in the experiments contains eight channels which include three phase currents, three phase voltages, $60-\mathrm{Hz}$ notch-filtered first phase current, and accelerometer data. Notch-filtered data were collected in anticipation of improving the dynamic range of the A/D converter. Accelerometer data were used in the bearing tests to validate the location of the fault frequencies.

\section{A. Bearing Fault Experiments}

The bearing fault experiments were performed using the first, sixth, and the eighth channels to evaluate the significance of notch filtering and the accelerometer data. The data were collected for about $40 \mathrm{~s}$, yielding 80000 points per channel. Later, these data sets were downsampled to study the effect of lower rate sampling. The length of the windowing function was selected so that the time-frequency spectrum has $0.2 \mathrm{~Hz} / \mathrm{bin}$ resolution, which resulted in eight contiguous frequency spectra for each data set.

Table I shows the data sets used during the training stage of the bearing fault detection algorithm. The data were collected at different loads and speed. The bearing $\mathrm{C} 2$ is a healthy ball bearing which was pressed on the motor shaft and then soaked with oil before it was installed. The motor was run continuously for about $5 \mathrm{~min}$ during the various tests. In one case, an artificial turn fault was induced to check if the algorithm would be able to distinguish between the turn faults and bearing faults. Note that, for the healthy modes, a nonzero label is used; the label 0 is reserved for the fault modes.

Table II shows the data sets acquired from the same motor with faulty bearings. The inner and outer race defects were simulated by mounting the faulty bearing in different configurations. In the first case, the ball bearing with a hole mounted toward the outer part of the rotor shaft induced a severe outer race defect. In the second case, the bearing mounted toward the inner part of the rotor shaft induced a mild outer race defect. Fig. 9 illustrates the time-frequency spectrum of the motor current with healthy bearings around the bearing frequency. Ideally, the estimated frequency has to be at the middle of the window. However, the true bearing frequency usually shows
TABLE II

Testing Data From a 3/4-HP Motor with Damaged Bearings

\begin{tabular}{|c|c|c|c|c|}
\hline Mode & File Name & Torque & Speed & $\begin{array}{c}\text { Bearing and } \\
\text { Data ID } \\
\end{array}$ \\
\hline 0 & H0995001.asc & $+0.339 \mathrm{~N}-\mathrm{m}$ & 1789 Rpm & $\mathrm{H} 1$ with hole out. ${ }^{* *}$ \\
\hline 0 & Ho995002 asc & $+1.378 \mathrm{~N}-\mathrm{m}$ & $1762 \mathrm{Rpm}$ & H1 with hole out. \\
\hline 0 & H0995003.asc & $+2.825 \mathrm{~N}-\mathrm{m}$ & $1720 \mathrm{Rpm}$ & $\mathrm{H} 1$ with hole out \\
\hline 0 & Ho995004.asc & $+1.356 \mathrm{~N}-\mathrm{m}$ & $1762 \mathrm{Apm}$ & $\mathrm{H} 1{ }^{*}$ hole out $\&$ turn fault. \\
\hline 0 & H0995005.asc & $+0.332 \mathrm{~N}-\mathrm{m}$ & $1790 \mathrm{Rpm}$ & $\mathrm{H} 1$ with hole out. \\
\hline 0 & H0995006.asc & $+1.367 \mathrm{~N}-\mathrm{m}$ & $1762 \mathrm{Rpm}$ & $\mathrm{H} 1$ with hole out. \\
\hline 0 & H0995007.asc & $+2.825 \mathrm{~N}-\mathrm{m}$ & $1719 \mathrm{Rpm}$ & $\mathrm{H} 1$ with hole out. \\
\hline 0 & I1595001 asc & $+1.356 \mathrm{~N}-\mathrm{m}$ & $1761 \mathrm{Rpm}$ & H1 with hole in. ${ }^{\star \star \star}$ \\
\hline 0 & 11595002.asc & $+2.825 \mathrm{~N}-\mathrm{m}$ & $1717 \mathrm{Rpm}$ & H1 with hole in. \\
\hline
\end{tabular}

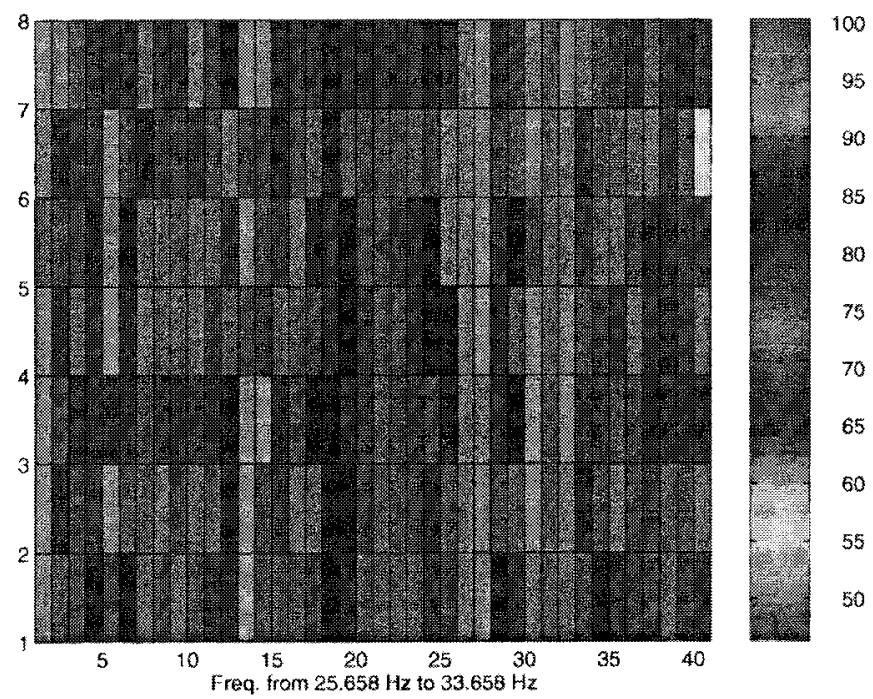

Fig. 9. Time-frequency spectrum of a normal mode.

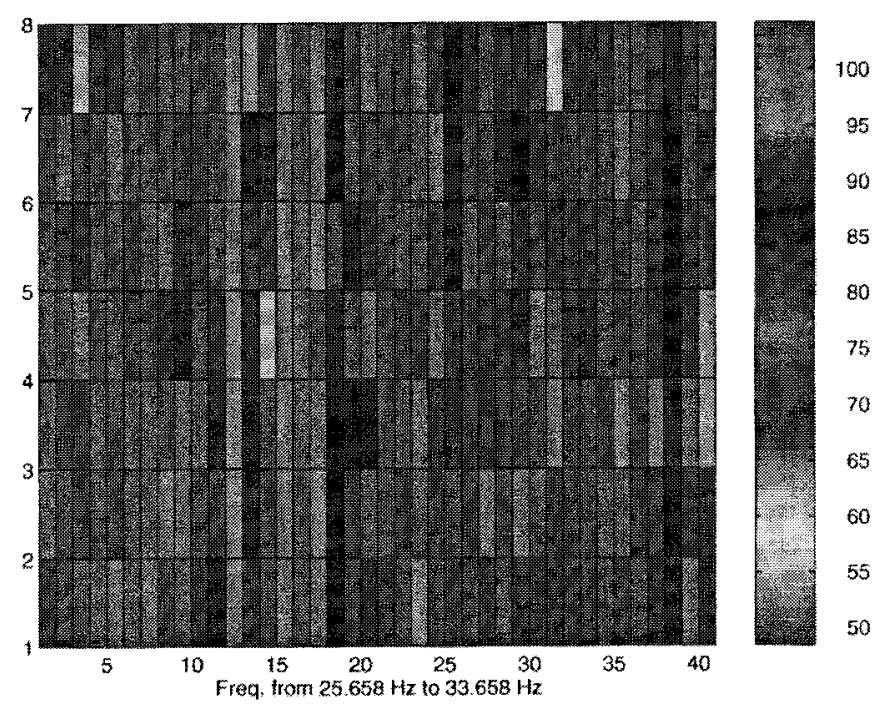

Fig. 10. Time-frequency spectrum of the motor with faulty bearings.

up 2-5 bins off the estimated frequency. Fig. 10 illustrates the time-frequency spectrum of the same motor with faulty bearings. In the case of faulty bearings, the bearing frequency 
TABLE III

Results of the Detection Tests for the Notch-Filtered Data Digitized at 32 Samples Per Power Supply

\begin{tabular}{l||c|c|c|c|c|c}
\hline Mode & 1 & 2 & 3 & 4 & 5 & 0 \\
\hline \hline 1 & 8 & & & & & \\
\hline 2 & & 7 & & & & 1 \\
\hline 3 & & & 8 & & & \\
\hline 4 & & & & 8 & & \\
\hline 5 & & & & & 7 & 1 \\
\hline 0 & & & & & & 72 \\
\hline
\end{tabular}

components are shifted downwards, since the bearing defect decreases the speed of the motor.

During the testing process, data from both Tables I and II were used. The training data were included in testing to validate the threshold selection criteria. The threshold was set as two unit standard deviations. Out of 112 samples from the sixth channel, 110 samples were correctly identified. Two samples from a normal operating mode with healthy bearings were misclassified as potential fault signals, yielding $98 \%$ accuracy and $2 \%$ false positive error. Samples from a normal operating mode were mostly within two standard deviations of the representatives. The false positive error is produced by those samples which are beyond two standard deviations. Nevertheless, these samples were still within at most 3.5 standard deviations of the representatives, which is very small as compared to the distance of even the closest fault signal. The detailed results of this test are tabulated in Table III. The diagonal entries in the matrix show the number of correctly classified samples and the off-diagonal entries show the number of misclassifications. For example, the entry at the $i$ th row and $j$ th column shows the number of samples from mode $i$ which are classified as mode $j$. In the case of perfect classification, the matrix becomes diagonal. The average distance of the first three fault data sets to the normal operating modes are shown in Table IV(a)-(c). These distances are at least in the order of tens. As we shall explain in the next section, these misclassifications can be easily avoided by analyzing the threshold setting further, which was arbitrarily chosen as two standard deviations. Therefore, the thresholds of the normal operating modes can be relaxed to reduce the rate of false positive error without decreasing the detection accuracy.

The experiments with the unfiltered data yielded the same result as the filtered data. In fact, visual inspection of feature vectors from both data sets did not reveal significant difference. The tests were repeated with the data downsampled to 12 samples per power cycle. The notch-filtered data resulted in $95 \%$ accuracy and $5 \%$ false positive rate. The unfiltered data resulted in $93 \%$ accuracy and $7 \%$ false positive rate. These results suggest that the proposed method does not require a high sampling rate.

Bearing experiments were also carried out with defective bearings of one, two, and three scratches on the outer race, one, two, and three scratches on the balls, and one, two, and three cage defects. All the defective measurements were correctly classified as defective.
TABLE IV

Average Distance of the Test Samples with Defective Bearings to the Normal Operating Modes

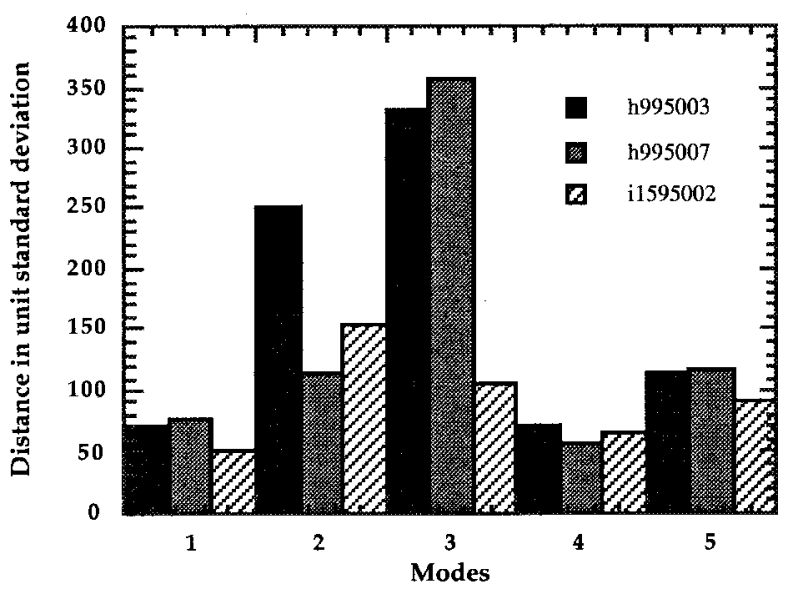

(a)

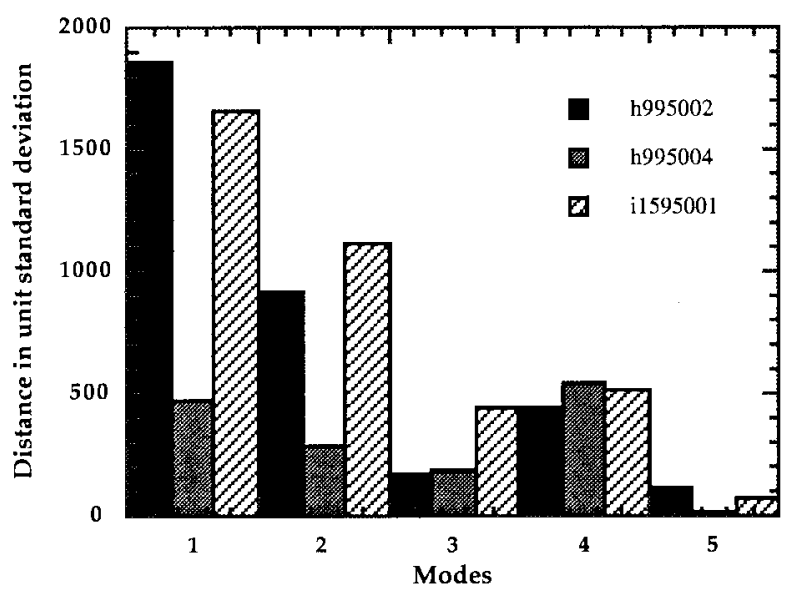

(b)

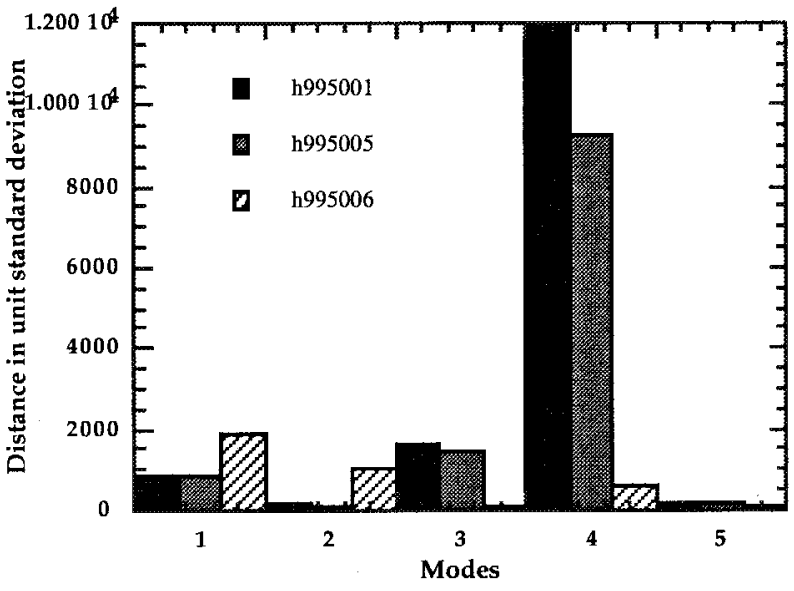

(c)

\section{B. Broken Bar Experiments}

For the training stage, a 35-hp inside-out motor operating in three different load and operating conditions was used. These data sets are tabulated in Table V. The time-frequency spectrum was computed so that the frequency resolution 
TABLE V

Training Data From the 35-hP MOtor with BroKen BAR

\begin{tabular}{c|c|c}
\hline \hline Mode & Torque & Alignment \\
\hline 1 & Over load & 10 mil offset \\
\hline 2 & Full load & Aligned \\
\hline 3 & No load & Aligned \\
\hline
\end{tabular}

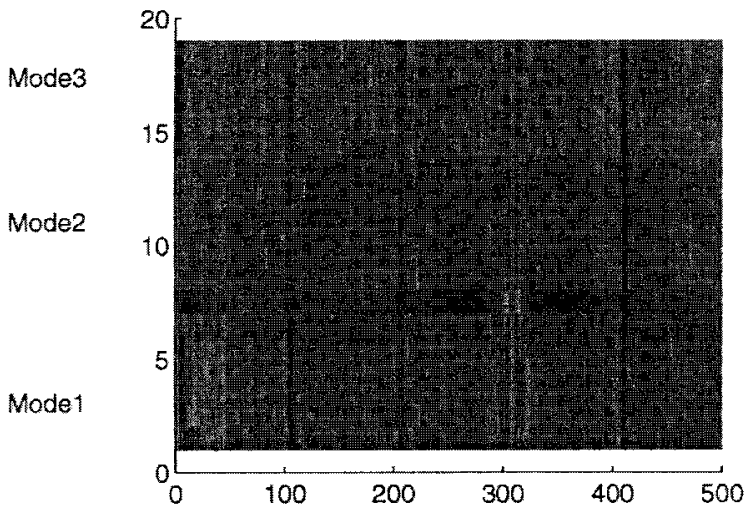

Fig. 11. Time-frequency spectrum of the normal operating modes of the broken bar motor.

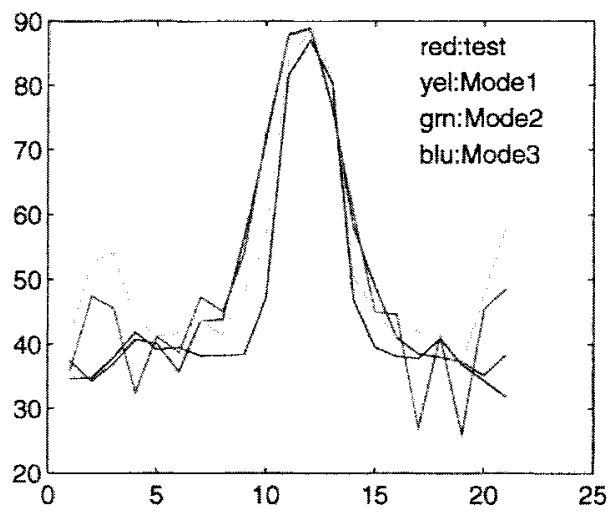

Fig. 12. The representatives of the normal operating modes of the broken bar motor around the fifth harmonic and a test feature from the motor with broken bars.

TABLE VI

Test Data From the 35-HP Motor With Broken Bars

\begin{tabular}{c|c|c|l}
\hline \hline Mode & Torque & Alignment & Broken Bars \\
\hline \hline 0 & No load & Aligned & 1 cut \\
\hline 0 & Full load & Aligned & 2 adjacent cuts \\
\hline 0 & No load & Aligned & 2 adjacent cuts \\
\hline
\end{tabular}

would be at least $0.2 \mathrm{~Hz} / \mathrm{bin}$. This yielded six strips of frequency spectra per data set. The time-frequency spectrum of the normal operating modes of the motor is shown in Fig. 11. The broken bar feature vector was formed by a window of frequency components around the first, fifth, and seventh harmonic sidebands, as described in Section IV-A. The representatives of the normal operating modes and a test feature around the fifth harmonic are shown in Fig. 12.
TABLE VII

Results of the Broken Bar Detection Tests for the Unfiltered Data Digitized at 32 Samples Per Power Cycle

\begin{tabular}{c||c|c|c|c}
\hline Mode & 1 & 2 & 3 & 0 \\
\hline \hline 1 & 6 & & & \\
\hline 2 & & 6 & & \\
\hline 3 & & & 6 & \\
\hline 0 & & & & 24 \\
\hline
\end{tabular}

In the testing stage, seven different sets of data, three of which were from a nondefective motor and four from motors with varying degrees of broken bars, load conditions, and rotating asymmetries were used. The specifics of these data sets are tabulated in Table VI. Out of 42 tests, all were correctly classified, resulting in $100 \%$ accuracy. The results are tabulated in detail in Table VII.

\section{CONCLUSIONS}

In this paper, we have discussed an adaptive time-frequency method to detect broken bar and bearing defects. It was shown that the time-frequency spectrum reveals the properties relevant to fault detection better than the Fourier spectrum in the transform domain. The method is based on a training approach in which all the distinct normal operating modes of the motor are learned before the actual testing starts. Our study suggests that segmenting the data into homogenous normal operating modes is necessary, because different operating modes exhibit different statistical properties due to nonstationary nature of the motor current. Overlooking this fact will deteriorate the performance of the detection. We showed that signals from faulty motors are several hundred standard deviations away from the normal operating modes, which indicates the power of the proposed statistical approach.

Also, we proposed new methods of estimating fault frequencies based on torque and mechanical speed estimations. We showed that a window of frequency components around the estimated fault frequencies has to be monitored, because the estimates, even in the case of exact knowledge of the motor geometry and the operating conditions, are never accurate. This approach also allows us to efficiently process frequency components which are spread due to low-frequency resolution.

Our study suggests that the proposed method is a mathematically general and powerful one which can be utilized to detect any fault that could show up in the motor current.

\section{ACKNOWLEDGMENT}

The authors would like to thank their colleagues at General Electric, R. Koegl and G. Robinson, for setting up the experiments and collecting the data, and Dr. W. Premerlani and Dr. A. Abdel-Malek for the valuable discussions.

\section{REFERENCES}

[1] G. B. Kliman and J. Stein, "Methods of motor current signature analysis," in Electric Machines and Power Systems, vol 20. New York: Hemisphere, pp. 463-474, 1992. 
[2] G. B. Kliman, "The detection of broken bars in motors," Electric Power Research Institute, Palo Alto, CA, Rep. GS-6589-L, Jan. 1990.

[3] J. Bertrand and P. Bertrand, "Time-frequency representation of broad band signals," in Proc. Int. Conf. Acoustics, Speech and Signal Processing (ICASSP'88), Apr. 11-14, 1988, vol. 4, pp. 2196-2199.

[4] B. Boashash, "Time-frequency signal analysis," in Advances in Spectrum Analysis and Array Processing, S. Haykin, Ed. Englewood Cliffs, NJ: Prentice-Hall, 1990, pp. 418-517.

[5] D. Gabor, "Theory of communications," Proc. Inst. Elect. Eng. vol. 93, pp. 429-457, 1946.

[6] G. B. Kliman et al., "Slip frequency determination for broken bar detectors," U.S. Patent 5049 815, Sept. 17, 1991.

[7] R. R. Schoen, B. K. Lin, T. G. Habetler, J. H. Schlag, and S. Farag, "An unsupervised, on-line system for induction motor fault detection using stator current monitoring," in Conf. Rec. 29th IEEE-IAS Annu. Meeting, Oct. 1994, pp. 117-122.

[8] R. R. Schoen, T. G. Habetler, F. Kamran, and R. G. Bartheld, "Motor bearing damage detection using stator current monitoring," in Conf. Rec. 29th IEEE-IAS Annu. Meeting, Oct. 1994, pp. 110-116.

[9] F. Flippetti, G. Franceschini, and C. Tassoni, "Neural networks aided on-line diagnostics of induction motor rotor failures," in Conf. Rec. 28th IEEE-IAS Annu. Meeting, Oct. 1993, pp. 316-323.

[10] G. B. Kliman, R. A. Koegl, W. J. Premerlani, and T. B. Breen, "Demonstartion of sensorless torque meter for AC motors," in Conf. Rec. 31st IEEE-IAS Annu. Meeting, Oct. 1996, pp. 633-637.

[11] W. Deleroi, "Broken bar in squirrel cage rotor of an induction motor-Part 1: Description by superimposed fault currents," Arch. Electrotech., vol. 67, pp. 91-99, 1984.

[12] G. B. Kliman et al., "Turn fault detection," U.S. Patent 5447 163, Dec. 1995.
[13] R. L. Schiltz, "Forcing frequency identification of rolling element bearings," Sound and Vibration, pp. 16-19, May 1990.

[14] An Introduction to Statistical Pattern Recognition, K. Fukunaga, Ed. New York: Academic, 1990.

[15] B. Yazoco and G. B. Kliman, "An unsupervised, on-line, method and apparatus for induction motor bearing fault detection using stator current monitoring," U.S. Patent 5726 905, Mar. 10, 1998.

[16] Pattern Recognition Principles, J. T. Tou and R. C. Gonzalez, Eds. Reading, MA: Addison-Wesley, 1974.

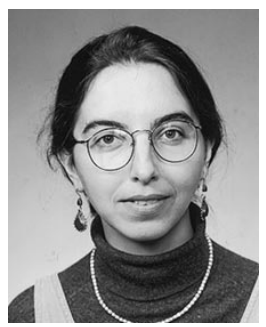

Birsen Yazıcı (S'92-M'95) received the B.S. degree in electrical engineering and mathematics from Bogazici University, Istanbul, Turkey, and the M.S. degree in mathematics and the Ph.D. degree in electrical engineering from Purdue University, West Lafayette, IN, in 1988, 1990, and 1994, respectively.

Since 1994, she has been with the Corporate Research and Development Center, General Electric Company, Niskayuna, NY. Her current research interests include applied mathematics, statistical signal processing, pattern recognition and condition monitoring, and fault detection in industrial systems.

Gerald B. Kliman (S'52-M'55-SM'76-F'92-LF'95), for a photograph and biography, see p. 99 of the January/February 1999 issue of this TRANSACTIONS. 